Technical Report No. 06/05, November 2005

ON THE EXPECTED DISCOUNTED PENALTY FUNCTION FOR LÉVY RISK PROCESSES

José Garrido and Manuel Morales 


\title{
On the Expected Discounted Penalty Function for Lévy Risk Processes*
}

\author{
José Garrido \\ Department of Mathematics and Statistics \\ Concordia University \\ and \\ Manuel Morales \\ Department of Mathematics and Statistics \\ University of Montreal
}

\begin{abstract}
Dufresne et al. (1991) introduced a general risk model defined as the limit of compound Poisson processes. Such a model is either a compound Poisson process itself or a process with an infinite number of small jumps. Later, in a series of now classical papers, they studied the joint distribution of the time of ruin, the surplus before ruin and the deficit at ruin [Gerber and Shiu (1997, 1998a, 1998b), Gerber and Landry (1998)]. They work with the classical and the perturbed risk models and hint that their results can be extended to gamma and inverse Gaussian risk processes.

In this paper we work out this extension in the context of a more general risk model. The construction of Dufresne et al. (1991) is based on a non-negative, non-increasing function $Q$ that governs the jumps of the process. This function, it turns out, is the tail of the Lévy measure of the process. Our aim is to extend their work to a generalized risk model driven by an increasing Lévy process. This first paper
\end{abstract}

*This research was funded by a Society of Actuaries (AERF/CKER project) and the Natural Sciences and Engineering Research Council of Canada (NSERC) operating grants 368601999 and 3116602005 . 
presents the results for the case when the aggregate claims process is a subordinator. Embedded in this wide family of risk models we

find the gamma, inverse Gaussian and generalized inverse Gaussian processes.

\section{Introduction}

We first discuss a risk model with a surplus process of the form

$$
U(t)=u+c t-S(t)+\eta Z(t), \quad t \geq 0,
$$

where $S$ is a subordinator with zero drift and Lévy measure $q$ and $Z$ is a Lévy motion with no positive jumps. $u$ is the initial surplus and $c$ is a constant premium rate defined as $c=(1+\theta) \mathbb{E}[S(1)]$, where $\theta$ is the security loading factor. For an account on the classical risk model we refer to Grandell (1991), Asmussen (2000) or Kaas et al. (2001).

Ruin probabilities in this model have been discussed in Bertoin and Doney (1994), Yang and Zhang (2001), Morales and Schoutens (2003), Huzak et al. (2004), Klüppelberg et al. (2004) and in Doney and Kyprianou (2005). The results on ruin probabilities for (1) follow from well known results in fluctuation theory for Lévy processes. Huzak et al. (2004) study a ladderheight decomposition for the ruin probability, Klüppelberg et al. (2004) and Doney and Kyprianou (2005) study the ruin probability asymptotics, as well as the over- and under-shoot for such a model. However, the Gerber-Shiu function has not yet been explored in such a context.

The Gerber-Shiu function was introduced in Gerber and Shiu (1998a) and was defined to capture several quantities of interest in risk theory, namely the ruin probability, the Laplace transform of the time to ruin and the joint density of the surplus prior to ruin and the deficit at ruin. Results for this function are embedded in the quintuple law discussed in Doney and Kyprianou (2005). It is of independent interest to derive and interpret these results in such a way that they remain compatible with the existing actuarial literature. It is also useful to explore examples for which computations can be carried out beyond the general expressions.

In this article we work out the extension of work by Dufresne, Gerber and Shiu for subordinators, a sub-class of Lévy processes. Unlike the classical case, that models the individual claim size distribution and obtains from it the aggregate distribution, here the aggregate claims distribution is known 
in closed form. In particular, Sections 3.2, 4.3 and 5 discuss examples for which the general results can be carried forward in more detail. This is a straight-forward application of the characterization of subordinators, as limits of compound Poisson processes. Nonetheless, it brings new insight to the applicability of Gerber-Shiu functions in general risk models.

We start by defining subordinators and reviewing their first properties.

\section{Subordinators}

All the stochastic processes in the paper are assumed to be defined on a filtered probability space $\left(\Omega, \mathcal{F},\left(\mathcal{F}_{t}\right)_{t \geq 0}, \mathbb{P}\right)$.

Subordinators form the subclass of increasing Lévy processes with paths of finite variation. Lévy processes are in a one-to-one correspondence with the class of infinitely divisible distributions [see Barndorff-Nielsen et al. (2001), Bertoin (1996) or Sato (1999) for accounts on Lévy processes and subordinators]. Their Laplace transform $\phi_{t}(s)=\mathbb{E}\left(e^{-s X(t)}\right)$ is of the form $e^{t \Psi(s)}$, where $\Psi$ is the so-called Laplace exponent in the Lévy-Khintchine characterization, as given in the following definition.

Definition 2.1 An adapted càdlàg $\mathbb{R}$-valued process $X=\{X(t)\}_{t \geq 0}$ with $X(0)=0$ is a Lévy process if its Laplace transform is of the form $\bar{\phi}_{t}(s)=$ $e^{t \Psi(s)}$, where

$$
\Psi(s)=-a s+\frac{b^{2}}{2} s^{2}-\int_{\mathbb{R}_{0}}\left[1-e^{-s x}-s x \mathbb{I}_{\{(-1,1)\}}(x)\right] q(d x), \quad s \in \mathbb{R},
$$

with $a, b \in \mathbb{R}$ and $q$ is a positive measure on $\mathbb{R}_{0}=\mathbb{R}-\{0\}$ satisfying

$$
\int_{\mathbb{R}_{0}}\left(1 \wedge|x|^{2}\right) q(d x)<\infty
$$

The parameters $a, b^{2}$ and $q$ uniquely determine $X$. They are referred to as the triplet of Lévy characteristics (or Lévy triplet for short) $\left[a, b^{2}, q(d x)\right]$. The measure $q$ is called the Lévy measure and the exponent $\Psi$ is called the Laplace exponent of the process $X$.

It is useful to define also the cumulant exponent of a Lévy process $\vartheta$. This is simply the exponent appearing in the moment generating function of 
the process, i.e. $\mathbb{E}\left(e^{s X(t)}\right)=e^{t \vartheta(s)}$. The cumulant exponent is clearly given by the Laplace exponent as $\vartheta(s)=\Psi(-s)$.

The Lévy measure governs the occurrence and the size of the jumps of the process $X$. For instance, the number of jumps of size larger than any $\epsilon>0$ is a Poisson process with mean $\bar{Q}(\epsilon)=\int_{\epsilon}^{\infty} q(d x)$, where $\bar{Q}=1-Q$ is the integrated tail of $q$. The density function of the jump sizes is then $\frac{q(d x)}{Q(\epsilon)} \mathbb{I}_{(\epsilon, \infty)}(x)$. Hence, the jumps larger than $\epsilon$ form a compound Poisson process.

If $b^{2}>0$ and the Lévy measure is identically zero then the process is a Brownian motion (the only continuous Lévy process). When the Gaussian coefficient $b^{2}=0$ the process is entirely composed by jumps, if in addition $\int_{\mathbb{R}_{0}} q(d x)<\infty$ then the process is a compound Poisson process, while if $\int_{\mathbb{R}_{0}} q(d x)=\infty$ and $\int_{\mathbb{R}_{0}}(1 \wedge|x|) q(d x)<\infty$, then the process has an infinite number of small jumps but is of finite variation. Finally, if $\int_{\mathbb{R}_{0}} q(d x)=\infty$ and $\int_{\mathbb{R}_{0}}(1 \wedge|x|) q(d x)=\infty$, the process has infinitely many jumps and is of unbounded variation.

If the Gaussian coefficient $b^{2}=0$ and the Lévy measure $q$ is defined on $(0, \infty)$, such that $\int_{0}^{\infty}(1 \wedge x) q(d x)<\infty$, then the corresponding Lévy process is called a subordinator. Its increments are always positive and moreover, its Laplace exponent in (2) can be written as

$$
\Psi(s)=-a s+\int_{0}^{\infty}\left(e^{-s x}-1\right) q(d x), \quad s>0 .
$$

This last equation characterizes the family of all subordinators. Alternatively, in terms of the integrated tail $\bar{Q}$ of the Lévy measure, we can rewrite (3) as [see Bertoin (1996)]:

$$
\frac{\Psi(s)}{s}=a+\int_{0}^{\infty} e^{-s x} \bar{Q}(x) d x, \quad s>0 .
$$

\subsection{Examples of subordinators}

Illustrative examples of subordinators are the $\alpha$-stable subordinator, the gamma process and the generalized inverse Gaussian process. 


\subsection{1 $\quad \alpha$-stable subordinator}

If the Laplace exponent given by

$$
\Psi(s)=s^{\alpha}=\frac{\alpha}{\Gamma(1-\alpha)} \int_{0}^{\infty}\left(1-e^{-s x}\right) x^{-1-\alpha} d x, \quad s>0,
$$

with $\alpha \in(0,1)$ then the process $X$ is called an $\alpha$-stable subordinator. The Lévy measure is given by

$$
q(d x)=\frac{\alpha}{\Gamma(1-\alpha)} x^{-1-\alpha} d x, \quad x>0 .
$$

Notice that $\bar{Q}(0)=\int_{0}^{\infty} q(d x)=\infty$ and therefore the process has infinitely many small jumps.

The $\alpha$-stable subordinator is a subclass of the larger family of $\alpha$-stable processes. The restriction on the parameter $0<\alpha<1$ is due to the condition $\int_{0}^{\infty}(1 \wedge x) q(d x)<\infty$. The increments of this process follow a positive $\alpha-$ stable distribution.

The $\alpha$-stable family is studied extensively in Janicki and Weron (1994). In insurance, the $\alpha$-stable process has been recently used for risk models in the presence of large claims [Furrer et al. (1997) and Furrer (1998)]. When $\alpha \in[1,2)$, the $\alpha$-stable process is no longer a subordinator, but it can be used as the Lévy perturbation in (1) (see Section 3).

\subsubsection{Gamma process}

The gamma process is a subordinator with Laplace exponent given by

$$
\Psi(s)=-a \ln \left(1+\frac{s}{b}\right)=\int_{0}^{\infty}\left(e^{-s x}-1\right) a x^{-1} e^{-b x} d x, \quad s>0,
$$

where $a, b>0$. Clearly the Lévy measure is given by

$$
q(d x)=a x^{-1} e^{-b x} d x, \quad x>0 .
$$

We can easily see that the mean of this process at time one is

$$
\mu_{X}=\mathbb{E}[X(1)]=a / b .
$$

Notice that $\bar{Q}(0)=\int_{0}^{\infty} q(d x)=\infty$ and therefore this process also has infinitely many small jumps. The increments of this process follow a gamma 


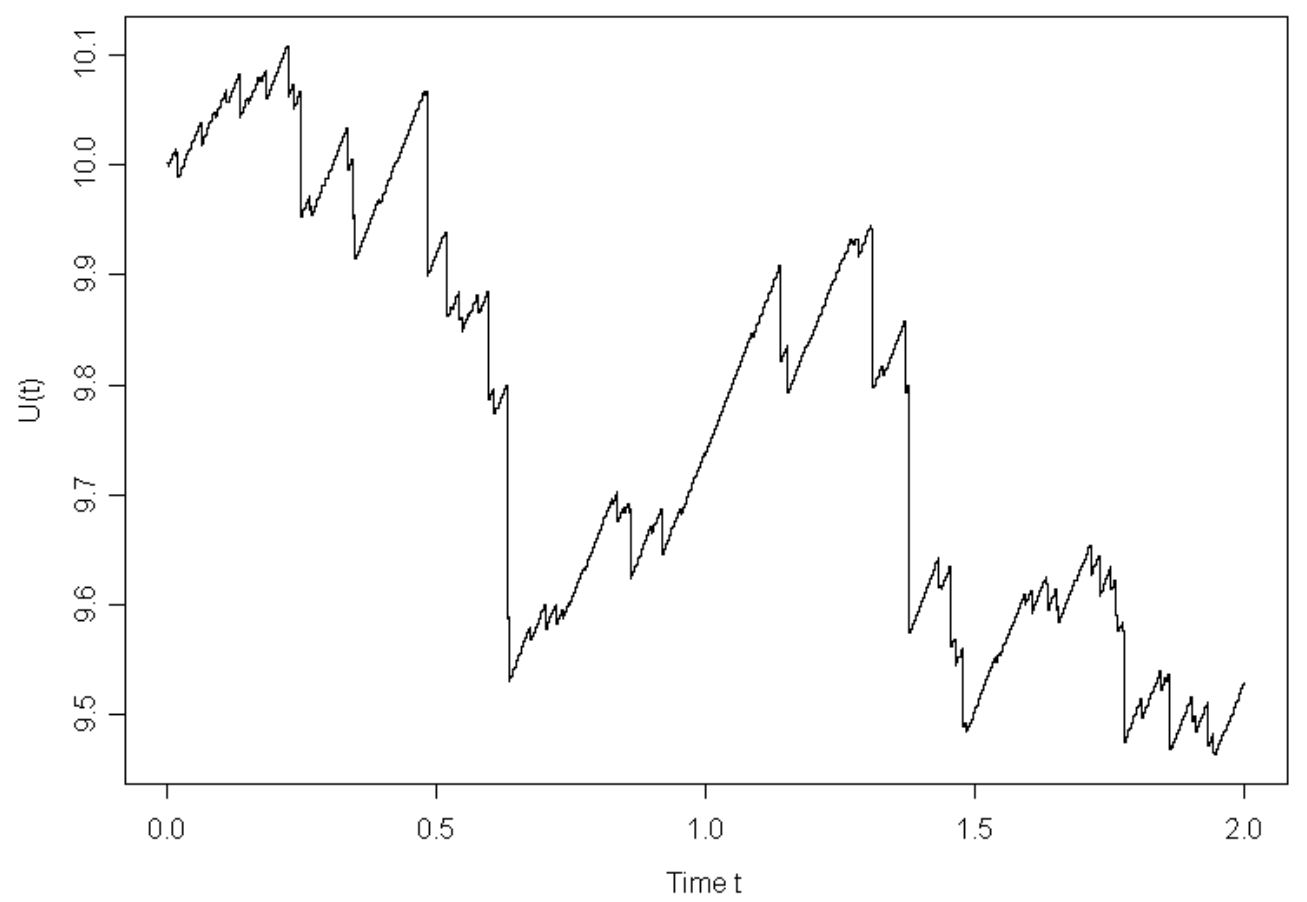

Figure 1: Surplus process in (1) with a gamma subordinator $(a=15, b=10)$

distribution; see the illustrative path in Figure 1 with initial surplus $u=10$, premium rate $c=1.3$, gamma parameters $a=15$ and $b=10$, but no Lévy perturbation $(\eta=0)$.

This process has been used in finance as well as in insurance [see Dufresne et al. (1991) and Madan et al. (1998)].

\subsubsection{Generalized inverse Gaussian process}

The class of generalized inverse Gaussian (GIG) distributions is characterized by three parameters: $\beta, \gamma$ and $\kappa$. If $\beta=-1 / 2$ the GIG distribution reduces to an inverse Gaussian. The gamma distribution is a limiting case of the GIG distribution for $\beta>0, \gamma>0$ and $\kappa \rightarrow 0$. These make the GIG Lévy processes a natural extention to the gamma process.

GIG distributions have been extensively studied by Jørgensen (1982). Barndorff-Nielsen and Halgreen (1977) showed that this family is infinitely divisible and therefore we can define a positive Lévy process. The generalized 
inverse Gaussian process is a subordinator with Laplace exponent given by

$$
\Psi(s)=\ln \left[\frac{K_{\beta}\left(\kappa \gamma \sqrt{1+\frac{2 s}{\gamma^{2}}}\right)}{K_{\beta}(\kappa \gamma)\left(1+\frac{2 s}{\gamma^{2}}\right)^{\beta / 2}}\right]=\int_{0}^{\infty}\left(e^{-s x}-1\right) q(d x),
$$

where $\beta \in \mathbb{R}, \kappa, \gamma>0$ and $K_{\beta}$ is the modified Bessel function of the third kind with index $\beta$. Its domain is $z>-\gamma^{2} / 2$ when $\beta \geqslant 0$, but $z \geqslant-\gamma^{2} / 2$ when $\beta<0$. Its Lévy measure $q$ is given by

$$
q(d x)=\frac{1}{x}\left[\kappa^{2} \int_{0}^{\infty} e^{-x t} g_{\beta}\left(2 \kappa^{2} t\right) d t+\max \{0, \beta\}\right] e^{-\gamma^{2} x / 2} d x
$$

where

$$
g_{\beta}(y)=\left\{\frac{\pi^{2}}{2} y\left[J_{|\beta|}^{2}(\sqrt{y})+N_{|\beta|}^{2}(\sqrt{y})\right]\right\}^{-1} .
$$

$J$ and $N$ are modified Bessel functions.

The mean of the process at time one is

$$
\mu_{X}=\mathbb{E}[X(1)]=\frac{\kappa}{\gamma} \frac{K_{1+\beta}(\kappa \gamma)}{K_{\beta}(\kappa \gamma)} .
$$

Note that $\bar{Q}(0)=\int_{0}^{\infty} q(d x)=\infty$ and the process is composed again of an infinite number of small jumps. The increments of length one of this process follow a generalized inverse Gaussian distribution.

A particular member of this family, the inverse Gaussian process, has been proven to be a good model for aggregate claims [Chaubey et al. (1998)]; see the illustrative path in Figure 2 with initial surplus $u=10$, premium rate $c=2$ and inverse Gaussian parameters $\beta=-1 / 2, \kappa=4$ and $\gamma=2$ (no Lévy perturbation, $\eta=0$ ).

A GIG family of processes has been recently proposed in insurance to model aggregate claims [see Morales (2004)].

\section{$3 \quad$ A general perturbed risk model}

\subsection{The model}

Consider a general perturbed risk model based on a subordinator for the aggregate claims and a spectrally negative Lévy process for the perturbation, 


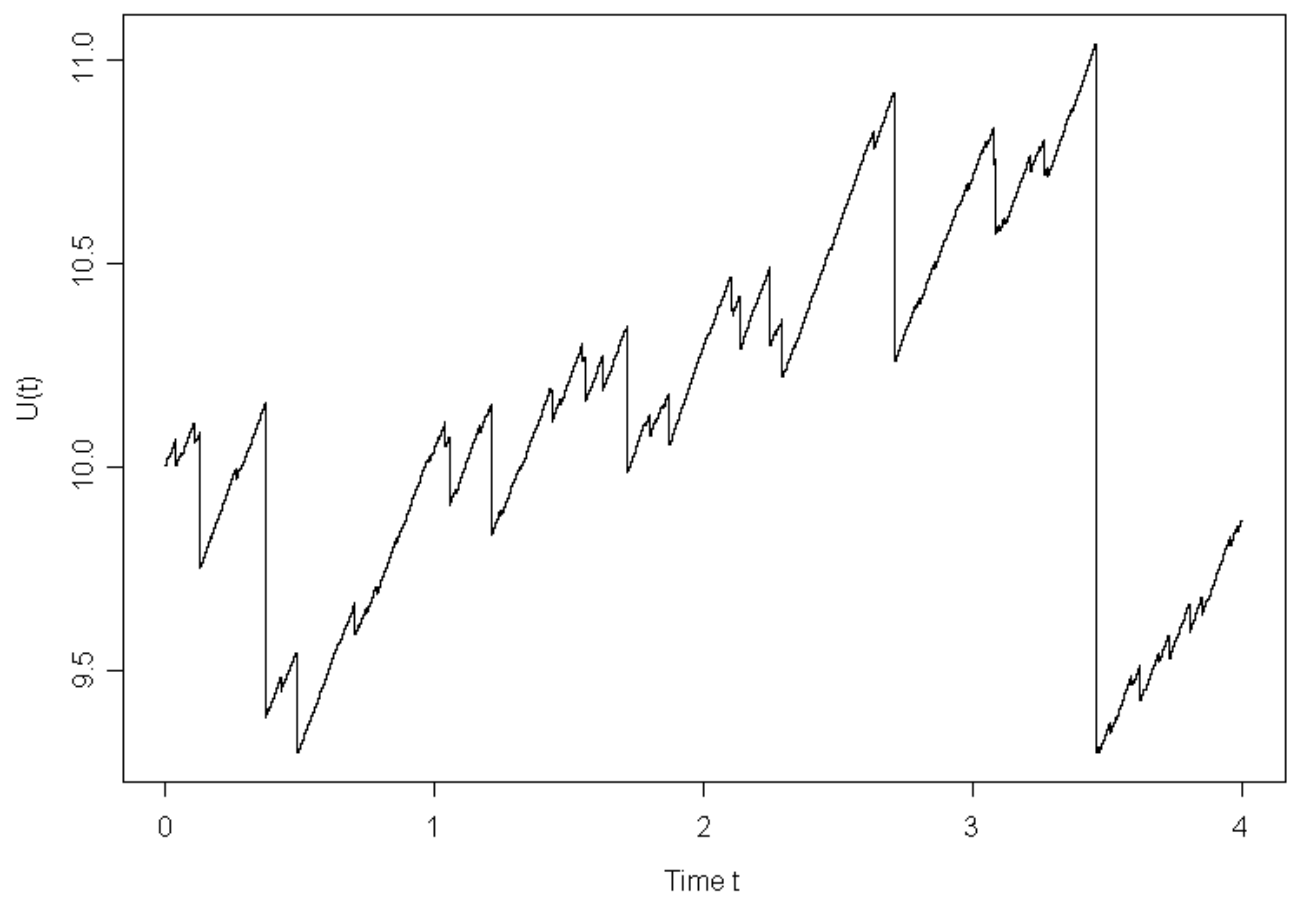

Figure 2: Surplus process in (1) with an inverse Gaussian subordinator $(\beta=$ $-1 / 2, \kappa=4, \gamma=2)$

as in (1). This is quite a general model as it includes, as particular cases, several models previously considered in the literature.

Subordinators have appealing features as models for aggregate claims. They are increasing jump processes that can naturally account for claims. Despite their counterintuitive property of allowing for an infinite number of small jumps, they still preserve the ladder-height structure of the classical risk model.

Using a subordinator in the model implies an infinitely divisible distribution for the aggregate claims. Some possible choices are gamma, inverse Gaussian or generalized inverse Gaussian. All these have closed-form densities and have been used previously as models for aggregate claims. This can be seen as an advantage over defining the individual claims distribution first; recall that in the classical case the aggregate claims distribution does not have a nice closed-form expression. 
Despite the loss of detail in subordinator models, we can recover an individual claim size distribution. Simply divide the infinite number of jumps into two types: those smaller than a certain threshold $\epsilon$, which we will associate with an extra source of randomness (perturbation), and those larger than $\epsilon$ that can be seen as claims with a density $\frac{\bar{Q}(x)-\bar{Q}(\epsilon)}{\bar{Q}(\epsilon)}=\frac{\int_{\epsilon}^{x} q_{\epsilon}(s) d s}{\int_{\epsilon}^{\infty} q_{\epsilon}(s) d s}$. The tail behavior of such laws depends on the tail behavior of the Lévy measure $q$ [see Klüppelberg et al. (2004)].

As for the perturbation, spectrally negative Lévy processes are relatively tractable, despite their infinite number of small jumps. Incorporating this type of Lévy process in the model accounts for extra sources of randomness, still keeping a similar but more general structure for the ladder-height decomposition.

This general perturbed model has been recently studied in the literature. Yang and Zhang (2001) proposes a model like (1) where $Z$ is a Brownian motion special case. They adapt existing results for Lévy processes to an insurance context. Using a theorem from Zolotarev (1964), they interpret the ascending ladder-height process as a classical ladder-height structure for the ruin probability.

Huzak et al. (2004) work with the general model (1) and give a general ladder-height decomposition of the ruin probability. This illustrates the fact that classical results in ruin theory are embedded in the fluctuation theory for spectrally negative Lévy processes.

Chiu and Yin (2005) study the last time that the risk process crosses level zero, as well as the duration of the ruin event. They also use a theorem from Zolotarev (1964).

Both papers above rely on the following result (reproduced here from Zolotarev (1964) for completeness).

Theorem 3.1 Let $X=\{X(t)\}_{t \geq 0}$ be a Lévy process with no positive jumps, cumulant exponent $\vartheta_{X}$ and Lévy measure $q$. Moreover, let it have a finite mean $\mathbb{E}[X(1)]=\mu_{X}=\int_{0}^{\infty} x q(d x) \geq 0$. Define $\psi(u)=\mathbb{P}\left[\sup _{t \geq 0}-X(t)>u\right]$ for $u \geq 0$. Then we have that:

$$
s \int_{0}^{\infty} e^{-s u} \psi(u) d u=\frac{\mu_{X} s}{\vartheta_{X}(s)}, \quad s>0 .
$$

$\psi(u)$, above, is associated with the ruin probability for the model in (1). Let $X(t)=U(t)-u$, then $\psi$ is the ruin probability for (1). Since $X$ has no positive 
jumps, Theorem 3.1 applies and a general ladder-height decomposition for $\psi$ can be derived. This is spelled out in the following theorem.

Theorem 3.2 (Huzak et al. (2004)) Let $U$ be a risk process as in (1) and denote by $X(t)=U(t)-u$. Then, its associated ruin probability $\psi(u)=$ $\mathbb{P}\left[\sup _{t \geq 0}\{-X(t)\}>0\right]$ satisfies the following equation:

$$
1-\psi(u)=\frac{\theta}{1+\theta} \sum_{n=0}^{\infty}\left(\frac{1}{1+\theta}\right)^{n} M^{* n} * G^{*(n+1)}(u)
$$

where $M$ is like a ladder-height distribution, with Laplace transform given by

$$
\xi_{M}(s)=\int_{0}^{\infty} e^{-s x} d M(x)=\frac{\Psi_{S}(s)}{\mu_{S} s}
$$

for $\mu_{S}=\mathbb{E}[S(1)]$ and $G$ is the distribution with Laplace transform given by

$$
\xi_{G}(s)=\int_{0}^{\infty} e^{-s x} d G(x)=\frac{c s}{\Psi_{c t+\eta Z}(s)} .
$$

Recall that $\bar{Q}$ is the tail of the Lévy measure of the subordinator in (1) and $\Psi_{c t+\eta Z}$ is the Laplace exponent of the process $U(t)-u+S(t)=c t+\eta Z(t)$.

We can recognize $\xi_{M}$ to be the Laplace transform of a distribution given in terms of the tail of the Lévy measure of the subordinator $S$. From (4) we see that

$$
\xi_{M}(s)=\frac{\Psi_{S}(s)}{\mu_{S} s}=\int_{0}^{\infty} e^{-s x} \frac{\bar{Q}(x)}{\int_{0}^{\infty} \bar{Q}(t) d t} d x=\int_{0}^{\infty} e^{-s x} \frac{\bar{Q}(x)}{\mu_{S}} d x, \quad s>0,
$$

which allows us to identify the ladder-height-like density as

$$
m(x)=\frac{\bar{Q}(x)}{\int_{0}^{\infty} \bar{Q}(t) d t} .
$$

Equation (13) implies that $1-\psi(u)$ follows a defective renewal equation of the form

$$
1-\psi(u)=\frac{M(u)}{1+\theta}+\frac{\theta}{1+\theta} \int_{0}^{u}[1-\psi(u-s)] d(M * G)(s), \quad u \geqslant 0
$$


where the operator $*$ is the convolution of functions on $\mathbb{R}^{+}$defined as $f *$ $g(x)=\int_{0}^{x} f(x-y) g(y) d y$. From (13), using standard renewal theory techniques [see for example Asmussen (2000)], we obtain (17).

Clearly this unifying approach to risk modelling through Lévy processes, not only brings new insight on some well known models, but also enlarges the class of risk processes. These two effects are illustrated in the following sections.

For instance, Theorem 3.1 gives a relationship between the ruin probability and the Laplace exponent of the risk process. This is used in Section 5.4 to calculate ruin probabilities by inverting Laplace transforms.

Theorem 3.2 gives a general decomposition for the ruin probability that expands the usual ladder-height interpretation. Here each step in the ladder can be decomposed into two independent random variables. This clearly separates the effect of the subordinator from that of the perturbation in each ladder step. The corresponding distributions of these random variables, $M$ and $G$, are given in terms of their Laplace transforms and are related to the Laplace exponents of the subordinator and the perturbation. This can be used in direct computation to obtain ruin probabilities in a larger class of processes. For instance, the methods described in Politis and Pitts (2005) could be adapted to this more general model.

\subsection{Examples}

The following illustrative examples will help understand the generality of the model in (1).

\subsubsection{Classical compound Poisson model}

The classical risk model is included in (1), when $S$ is a compound Poisson process and there is no perturbation, i.e.

$$
U(t)=u+c t-S(t), \quad t \geq 0,
$$

where the jump distribution is $F$, say. We can see that equation (17) reduces to the known renewal equation for the ruin probability while its solution (13) is the well-known Beekman's convolution formula [see Bowers et al. (1997)].

Since there is no perturbation here, we also see that the Laplace transform $\xi_{G}$ is constant at 1 , hence recovering the Laplace transform of a compound geometric. The jumps in such a compound random variable are given by 
(16), which reduces to the so-called ladder-height transformation of the claim distribution $F$.

\subsection{2 $\alpha$-stable perturbation}

Furrer (1998) studies the model in (1) for a compound Poisson process $S$ and $Z=Z_{\alpha}$ an $\alpha$-stable Lévy process with no positive jumps, i.e.

$$
U(t)=u+c t-S(t)+\eta Z_{\alpha}(t), \quad t \geq 0 .
$$

The compound Poisson process has a jump distribution $F$. Furrer shows that the function $\xi_{G}$ of the decomposition in (13), is the Laplace transform of a distribution function $G$ given by

$$
G(x)=1-\sum_{n=0}^{\infty} \frac{\left(-c / \eta^{\alpha}\right)^{n}}{\Gamma(1+(\alpha-1) n)} x^{(\alpha-1) n}, \quad x \geq 0 .
$$

Hence the renewal equation (17) and its solution (13) hold with the above $G$, with the ladder-height transformation $M$ of the jump distribution $F$ :

$$
M(x)=\frac{1}{\beta} \int_{0}^{x}[1-F(t)] d t, \quad x>0,
$$

and where $\beta=\int_{0}^{\infty}[1-F(x)] d x<\infty$ is the mean jump.

\subsubsection{Brownian motion perturbation}

Dufresne and Gerber (1991) studies model (1) when $Z=W$ is a Brownian motion with zero drift and infinitesimal variance $\sigma^{2}$, and $S$ is a compound Poisson process, i.e.

$$
U(t)=u+c t-S(t)+\sigma W(t), \quad t \geq 0 .
$$

This is a particular case of the model of Furrer (1998), since a Brownian motion is an $\alpha$-stable Lévy process with index $\alpha=2$. Equations (13) and (17) hold with $M$ given by (19). As for the function $G$ in (18), it reduces to

$$
G(x)=1-e^{-\frac{2 c}{\sigma^{2}} x}, \quad x>0,
$$

i.e. an exponential distribution. 


\subsubsection{Gamma}

Dufresne et al. (1991) studies model (1) when $S$ is a gamma process and the perturbation $Z$ is not present, i.e.

$$
U(t)=u+c t-S(t), \quad t \geq 0 .
$$

If the aggregate process is a gamma process, then equation (13) takes the following form

$$
1-\psi(u)=\frac{\theta}{1+\theta} \sum_{n=0}^{\infty}\left(\frac{1}{1+\theta}\right)^{n} M^{* n}(u), \quad u \geq 0
$$

where $M$ is the distribution function of $m$ in (16), that is

$$
\begin{aligned}
M(x) & =\frac{\int_{0}^{x} \bar{Q}(t) d t}{\int_{0}^{\infty} \bar{Q}(t) d t} \\
& =b \int_{0}^{x} E_{1}(b t) d t=1-e^{-b x}+b x E_{1}(b x), \quad x \geq 0
\end{aligned}
$$

and where $E_{1}(x)=\int_{x}^{\infty} t^{-1} e^{-t} d t$ is the exponential integral function [see Abramowitz and Stegun (1970)].

This last equation comes from the fact that, for a gamma process with Lévy measure given by (7), we have

$$
\int_{0}^{\infty} \bar{Q}(t) d t=\int_{0}^{\infty} t q(d t)=\frac{a}{b}
$$

while

$$
\int_{0}^{x} \bar{Q}(t) d t=\int_{0}^{x} \int_{t}^{\infty} a s^{-1} e^{-b s} d s d t=a \int_{0}^{x} E_{1}(b t) d t, \quad \text { for } x \geq 0 .
$$

Notice that (20) implies that $\psi$ satisfies the renewal equation in (17):

$$
\psi(u)=\frac{1-M(u)}{1+\theta}+\frac{1}{1+\theta} \int_{0}^{u} \psi(u-y) d M(y), \quad u \geq 0 .
$$

In other words, (17) simplifies here to

$$
\psi(u)=\frac{e^{-b x}-b x E_{1}(b x)}{1+\theta}+\frac{1}{1+\theta} \int_{0}^{u} \psi(u-y) b E_{1}(b t) d t, \quad u \geq 0,
$$

where $E_{1}$ is as in (22). 


\subsubsection{Generalized inverse Gaussian}

In Morales (2004) we find a model like (1) where $S$ is a generalized inverse Gaussian Lévy process, without the perturbation $Z$, i.e.

$$
U(t)=u+c t-S(t), \quad t \geq 0 .
$$

In this case, (13) takes the following form

$$
1-\psi(u)=\frac{\theta}{1+\theta} \sum_{n=0}^{\infty}\left(\frac{1}{1+\theta}\right)^{n} M^{* n}(u), \quad u \geq 0
$$

where $M$ is as in (21) and $\bar{Q}$ is the tail of the Lévy measure of a GIG process, given in (10). This expression has to be computed numerically.

However, if the parameter $\beta$ of the GIG is $\pm 1 / 2$ then closed forms for $M$ exist since the function $g_{\beta}$ in (10) becomes $g_{\beta}(y)=\frac{1}{\pi \sqrt{y}}$. In particular, if $\beta=1 / 2, M$ becomes

$$
\begin{aligned}
M(x)=1 & -\frac{\gamma^{2}}{\kappa \gamma+1}\left\{\frac{2 \kappa \Gamma\left(\frac{1}{2}\right)}{\pi \gamma} \bar{\Gamma}\left(\frac{\gamma^{2}}{2} x ; \frac{1}{2}\right)-\frac{\kappa \Gamma\left(\frac{1}{2}\right)}{\pi}\left[\frac{2}{\gamma} \bar{\Gamma}\left(\frac{\gamma^{2}}{2} x ; \frac{3}{2}\right)\right.\right. \\
& \left.\left.-\gamma x \bar{\Gamma}\left(\frac{\gamma^{2}}{2} x ; \frac{1}{2}\right)\right]+\frac{1}{2}\left[\frac{2}{\gamma^{2}} e^{-\frac{\gamma^{2}}{2} x}-x \bar{\Gamma}\left(\frac{\gamma^{2}}{2} x ; 0\right)\right]\right\},
\end{aligned}
$$

where $\bar{\Gamma}(u ; \alpha)=\int_{u}^{\infty} x^{\alpha-1} e^{-x} d x$ is the tail of the usual incomplete gamma function. Note that $\bar{\Gamma}(u ; 0)$ is also the exponential integral function $-E_{1}(-u)$ in $(22)$.

If $\beta=-1 / 2$ (inverse Gaussian process) then $M$ is given by

$$
\begin{aligned}
M(x)= & 1-\frac{\gamma}{\kappa}\left\{\frac{2 \kappa \Gamma\left(\frac{1}{2}\right)}{\pi \gamma} \bar{\Gamma}\left(\frac{\gamma^{2}}{2} x ; \frac{1}{2}\right)-\frac{\kappa \Gamma\left(\frac{1}{2}\right)}{\pi}\left[\frac{2}{\gamma} \bar{\Gamma}\left(\frac{\gamma^{2}}{2} x ; \frac{3}{2}\right)\right.\right. \\
& \left.\left.-\gamma x \bar{\Gamma}\left(\frac{\gamma^{2}}{2} x ; \frac{1}{2}\right)\right]\right\} .
\end{aligned}
$$

This last expression comes from the fact that, for a GIG process with Lévy measure given by (10), we have

$$
\int_{0}^{\infty} \bar{Q}(t) d t=\int_{0}^{\infty} t q(d t)=\frac{\kappa \gamma+1}{\gamma^{2}} .
$$

Note that (25) implies that $\psi$ satisfies a renewal equation in terms of the function $M$. For instance, in the case where $\beta=1 / 2$, equation (23) can be 
written in terms of $M$, in (26), or its corresponding density function $m$ given by [see Morales (2004) for details]:

$$
m(x)=\left[\frac{\kappa \Gamma\left(\frac{1}{2}\right)}{\pi \sqrt{2}} \int_{x}^{\infty} t^{-\frac{3}{2}} e^{-\frac{\gamma^{2}}{2} t} d t+\frac{1}{2} \int_{x}^{\infty} t^{-1} e^{-\frac{\gamma^{2}}{2} t} d t\right], \quad x \geq 0
$$

\section{Expected discounted penalty function}

Gerber and Shiu (1998a) introduces the concept of expected discounted penalty function as a way to study the distribution of the time to ruin, the surplus at and prior to ruin. For the classical risk model, the expected discounted penalty function $\phi$, henceforth called the Gerber-Shiu (G-S) function, is defined as follows:

$$
\phi(u)=\mathbb{E}\left[w(U(\tau-),|U(\tau)|) e^{-\delta \tau} \mathbb{I}_{\{\tau<\infty\}} \mid U(0)=u\right], \quad u \geq 0,
$$

where $w(x, y)$ is a non-negative penalty function for being ruined, $u$ the initial surplus, $\tau$ is the time of ruin and $U(\tau-)$ the surplus just prior to ruin.

Gerber and Shiu show that (28) can be written as a convolution series:

$$
\phi(u)=h * \sum_{k=0}^{\infty} g^{* k}(u), \quad u \geq 0,
$$

for some functions $h$ and $g$.

The $\mathrm{G}-\mathrm{S}$ function can be extended to more general risk processes like (1). This problem will be worked out in its full generality in a sequel to this paper. We first study (1) without the perturbation $Z$, i.e. $\eta=0$ and hence

$$
U(t)=u+c t-S(t), \quad t \geq 0
$$

where $S$ is a subordinator.

Appendix A.1 shows that, for the risk process driven by a subordinator $S$ only $(\eta=0)$, the $\mathrm{G}-\mathrm{S}$ function in (28) satisfies (29) with

$$
g(x)=\frac{1}{1+\theta} \int_{x}^{\infty} e^{-\rho(y-x)} \frac{q(y)}{\int_{0}^{\infty} \bar{Q}(t) d t} d y, \quad x \geq 0 .
$$

and

$$
h(x)=\frac{1}{1+\theta} \int_{x}^{\infty} \int_{0}^{\infty} e^{-\rho(z-x)} w(z, y) \frac{q(z+y)}{\int_{0}^{\infty} \bar{Q}(t) d t} d y d z, \quad x \geq 0
$$


Here $q$ and $\bar{Q}$ are, respectively, the Lévy measure of the subordinator $S$ in (30) and its corresponding integrated tail. As for the constant $\rho$, it is the non-negative solution of the equation

$$
-\delta+c r-\Psi(r)=0, \quad r \geq 0,
$$

where $\Psi$ is the Laplace exponent of the subordinator in (30).

Remark 4.1 Note that equation (29) implies that the $G-S$ function is the solution of the equation

$$
\phi(z)=\int_{0}^{z} \phi(x) g(z-x) d x+h(z), \quad z>0,
$$

where the functions $g$ and $h$ are as in (31) and (32) respectively.

Remark 4.2 Denote by $\widehat{f}$ the Laplace transform $\widehat{f}(s)=\int_{0}^{\infty} e^{-s x} f(x) d x$ of a function $f$, for $s \geq 0$. Then the solution $\phi$ of (34) can be expressed also in terms of its Laplace transform $\widehat{\phi}$ :

$$
\widehat{\phi}(s)=\widehat{\phi}(s) \widehat{g}(s)+\widehat{h}(s)=\sum_{k=0}^{\infty}[\widehat{g}(s)]^{k} \widehat{h}(s)=\frac{\widehat{h}(s)}{1-\widehat{g}(s)}, \quad s \geq 0 .
$$

For subordinators, the structure of the Gerber-Shiu function is preserved and related results follow in the same straight-forward way. For example, if $\delta=0$, then $\rho=0$ and the differential term

$$
g(y) d y=\frac{1}{1+\theta} \frac{\bar{Q}(y)}{\int_{0}^{\infty} \bar{Q}(x) d x} d y=\frac{1}{1+\theta} m(y) d y,
$$

can be interpreted as the probability that the surplus will ever fall below $u$ and be between $u-y$ and $u-y-d y$. Recall that $m$ is the ladder-height density for the subordinator $S$.

Also if $\delta=0$ and $w(x, y)=1$, then

$$
h(x)=\frac{1}{1+\theta} \int_{x}^{\infty} m(t) d t=\frac{1}{1+\theta} \bar{M}(x),
$$

which can be interpreted as the probability that the surplus will ever fall below $u$ and will be below $u-x$ when it happens for the first time.

Other results that can be obtained by approaching risk models from the theory of Lévy processes. For example, a relation between the G-S function $\phi$ for a risk process driven by a subordinator and the ruin probability function $\psi$ is derived in the following result (see Appendix A.2 for the proof). 
Proposition 4.1 Let $\phi$ be the expected discounted penalty function as defined in (28) for a risk process driven by a subordinator $S$ with Lévy measure $q$. Let also $g$ and $h$ be the functions defined in (31) and (32), respectively. Then, the Laplace transform of $\phi$ is given by the following product

$$
\hat{\phi}(u)=\frac{1+\theta_{\rho}}{\theta_{\rho}} \hat{h}(u) \hat{\eta}(u), \quad u \geq 0
$$

where

$$
\hat{\eta}(s)=\frac{\frac{\theta_{\rho}}{1+\theta_{\rho}}}{1-\frac{1}{1+\theta_{\rho}} \hat{\psi}_{M_{\rho}}(s)},
$$

is the Laplace transform of the tail of a compound geometric distribution. Moreover, this distribution is the ruin probability $\psi_{\rho}$ of a risk process driven by a subordinator $S_{\rho}$ (the $-\rho$-Esscher transform of the original subordinator $S$ ) and loading factor $\theta_{\rho}=\frac{(1+\theta) \mu_{S}}{\mu_{\rho}}-1$, where $\mu_{\rho}=\mathbb{E}\left[S_{\rho}(1)\right]$.

This representation of the Gerber-Shiu function in terms of ruin probabilities of an Esscher-transformed risk process seems to be new. It can be compared to a similar expression derived in Drekic et al. (2004) for the classical risk model. Hence (35) should extend to subordinators their method to compute moments for the time of ruin.

\subsection{Ruin probabilities}

If $w(x, y)=1$ and $\delta=0$, then $\rho=0$ and the $\mathrm{G}-\mathrm{S}$ function reduces to the ultimate ruin probability, i.e. the renewal equation in (34) becomes

$$
\psi(u)=\frac{1-M(u)}{1+\theta}+\frac{1}{1+\theta} \int_{0}^{u} \psi(u-y) d M(y), \quad u \geq 0,
$$

Recall that $m$ and $M$ are, respectively, the ladder-height density and distribution defined in (16) and (21). Also, for $w(x, y)=1$ and $\delta=0,(31)$ and (32) become, respectively,

$$
g(x)=\frac{1}{1+\theta} \int_{x}^{\infty} \frac{q(y)}{\int_{0}^{\infty} \bar{Q}(t) d t} d y=\frac{1}{1+\theta} \frac{\bar{Q}(x)}{\int_{0}^{\infty} \bar{Q}(t) d t}=\frac{m(x)}{1+\theta}, \quad x \geq 0,
$$

and

$h(x)=\frac{1}{1+\theta} \int_{x}^{\infty} \int_{0}^{\infty} \frac{q(z+y)}{\int_{0}^{\infty} \bar{Q}(t) d t} d y d z=\int_{x}^{\infty} g(t) d t=\frac{1-M(x)}{1+\theta}, \quad x \geq 0$. 
Ruin probabilities can be computed numerically using (36), as its solution is a convolution series. Alternatively, Section 5 gives examples that use the relation between ruin probabilities and the Laplace exponent in Theorem 3.1. A numerical inversion of the Laplace transform then gives the ruin probabilities.

\subsection{Joint distribution of the surplus prior and at ruin}

Consider the discounted density function

$$
f_{\delta}(x, y \mid u)=\int_{0}^{\infty} e^{-\delta t} f(x, y, t \mid u) d t
$$

where $f(x, y, t \mid u)$ is the joint density of the surplus prior to ruin $U(\tau-)$, the deficit at ruin $|U(\tau)|$ and the time to ruin $\tau$. Note that $f_{\delta}$ can be recovered from the $\mathrm{G}-\mathrm{S}$ function when $w$ is an indicator function assigning value one to the point $\left(x_{0}, y_{0}\right)$, i.e. $\phi(u)=f_{\delta}\left(x_{0}, y_{0} \mid u\right)$ for $w(x, y)=\mathbb{I}_{\left(x_{0}, y_{0}\right)}(x, y)$.

Also note that if $\delta=0$, then $f_{\delta}\left(x_{0}, y_{0} \mid u\right):=f\left(x_{0}, y_{0} \mid u\right)$ is simply the joint density of the surplus prior to and the deficit at ruin. This implies that $f_{\delta}(x, y \mid u)$, and therefore $f(x, y \mid u)$, both follow a renewal equation of the form in $(34)$, i.e.

$$
f_{\delta}(x, y \mid u)=\int_{0}^{u} f_{\delta}(x, y \mid z) g(u-z) d z+h(u), \quad u>0
$$

where $g$ is given in (31) and $h$ in (32) simplifies to

$$
h(z)=\frac{1}{1+\theta} e^{-\rho(x-z)} \frac{q(x+y)}{\int_{0}^{\infty} \bar{Q}(t) d t} \mathbb{I}_{\{x>z\}}(z), \quad z \geq 0 .
$$

Hence, the solution of (37) is as follows:

$$
f_{\delta}(x, y \mid u)=h * \sum_{k=0}^{\infty} g^{* k}(u), \quad u \geq 0 .
$$

Classical results for the discounted joint density $f_{\delta}(x, y \mid 0)$ and its marginals can also be extended to the model (30) in a straight-forward way.

From (37) we have that $\phi(0)=h(0)$ and hence

$$
f_{\delta}(x, y \mid 0)=\frac{1}{1+\theta} e^{-\rho x} \frac{q(x+y)}{\int_{0}^{\infty} \bar{Q}(t) d t}, \quad x>0, y>0 .
$$


Integrating this function over $x$ gives the discounted marginal of the deficit at ruin:

$$
f_{\delta, 2}(y \mid 0)=\int_{0}^{\infty} f_{\delta}(x, y \mid 0) d x=g(y) .
$$

Similarly, the discounted marginal of the surplus before ruin is given by

$$
f_{1, \delta}(x \mid 0)=\int_{0}^{\infty} f_{\delta}(x, y \mid 0) d y=\frac{1}{1+\theta} e^{-\rho x} m(x) .
$$

Finally, the Laplace transform of the time to ruin $\tau$ is

$$
\begin{aligned}
\mathbb{E}\left[e^{-\delta \tau} \mathbb{I}_{\{\tau<\infty\}} \mid U(0)=0\right] & =\int_{0}^{\infty} \int_{0}^{\infty} f_{\delta}(x, y \mid 0) d y d x \\
& =\frac{1}{1+\theta} \int_{0}^{\infty} e^{-\rho x} m(x) d x
\end{aligned}
$$

\subsection{Examples}

The above results are illustrated with the gamma and generalized inverse Gaussian risk models of Section 3.2.

\subsubsection{Gamma process}

If the aggregate claims form a gamma process in (30), then the $\mathrm{G}-\mathrm{S}$ function (29) is the solution of the renewal equation in (34), with the functions $g$ and $h$ in (31) and (32), respectively. The latter simplify when the Lévy measure of a gamma process in (6) is used:

$$
g(x)=\frac{1}{1+\theta} \int_{x}^{\infty} e^{-\rho(y-x)} b y^{-1} e^{-b y} d y, \quad x \geq 0,
$$

and for $x \geq 0$,

$$
h(x)=\frac{1}{1+\theta} \int_{x}^{\infty} \int_{0}^{\infty} e^{-\rho(z-x)} w(z, y) b(z+y)^{-1} e^{-b(z+y)} d y d z,
$$

where $\rho$ is given by (33), which is here the non-negative solution of

$$
-\delta+c r+a \ln \left(1+\frac{r}{b}\right)=0 .
$$


These simplifications are due to the fact that for the gamma process $\int_{0}^{\infty} \bar{Q}(d t)=$ $\int_{0}^{\infty} t q(d t)=\frac{a}{b}$.

Setting $w(x, y)=1$ and $\delta=0$ to obtain ruin probabilities, yields $\rho=0$ and equations (40) and (41) become

$$
g(x)=\frac{1}{1+\theta} \int_{x}^{\infty} b y^{-1} e^{-b y} d y=\frac{1}{1+\theta} b E_{1}(b x)
$$

and

$$
h(x)=\frac{1}{1+\theta} \int_{x}^{\infty} g(y) d y=b x E_{1}(b x)-e^{-b x}
$$

respectively.

The $\mathrm{G}-\mathrm{S}$ function then gives the same ultimate ruin probability as in (24). Note that the resulting renewal equation for the ultimate ruin probability is also consistent with (23).

Similarly, the discounted joint density $f_{\delta}(x, y \mid u)$ satisfies a renewal equation of the form in $(37)$, i.e.

$$
f_{\delta}(x, y \mid u)=\int_{0}^{u} f_{\delta}(x, y \mid z) g(u-z) d z+h(u), \quad u>0
$$

where $g$ is given by (40) and

$$
h(z)=\frac{1}{1+\theta} e^{-\rho(x-z)} b(x+y)^{-1} e^{-b(x+y)} \mathbb{I}_{\{x>z\}}(z), \quad z \geq 0 .
$$

Finally, (38) and (39) show that for the gamma process

$$
f_{\delta, 2}(y \mid 0)=\frac{e^{\rho y}}{1+\theta} \int_{y}^{\infty} b x^{-1} e^{-(b+\rho) x} d x=\frac{b e^{\rho y}}{1+\theta} E_{1}[(b+\rho) y], \quad y>0,
$$

while the corresponding discounted marginal of the surplus before ruin is given by

$$
f_{1, \delta}(x \mid 0)=\frac{1}{1+\theta} e^{-\rho x} m(x)=\frac{e^{-\rho x}}{1+\theta} b E_{1}(b x), \quad x>0 .
$$

\subsubsection{Generalized inverse Gaussian}

If the aggregate claims in (30) form a generalized inverse Gaussian process, then the G-S function (29) is the solution of the renewal equation in (34), 
where the functions $g$ and $h$ are given by (31) and (32). The latter simplify when the Lévy measure of the GIG process in (10) is used. Simpler expressions are obtained when $\beta= \pm 1 / 2$.

For example, if $\beta=1 / 2$, then (31) and (32) take these forms, for $x \geq 0$ :

$$
g(x)=\frac{1}{1+\theta} \int_{x}^{\infty} e^{-\rho(y-x)} \frac{\gamma^{2}}{(\kappa \gamma+1)}\left[\frac{\kappa \Gamma(1 / 2)}{\pi \sqrt{2 y}}+\frac{1}{2}\right] y^{-1} e^{-\gamma^{2} y / 2} d y,
$$

and

$$
\begin{aligned}
h(x)= & \frac{1}{1+\theta} \int_{x}^{\infty} \int_{0}^{\infty} e^{-\rho(z-x)} w(z, y) \frac{\gamma^{2}}{(\kappa \gamma+1)}\left[\frac{\kappa \Gamma(1 / 2)}{\pi \sqrt{2(z+y)}}+\frac{1}{2}\right] \\
& \times(z+y)^{-1} e^{-\gamma^{2}(z+y) / 2} d y d z, \quad x \geq 0
\end{aligned}
$$

where $\rho$ is given by (33) and is here the non-negative solution of

$$
-\delta+c r-\ln \left[\frac{K_{\beta}\left(\kappa \gamma \sqrt{1+\frac{2 r}{\gamma^{2}}}\right)}{K_{\beta}(\kappa \gamma)\left(1+\frac{2 r}{\gamma^{2}}\right)^{\beta / 2}}\right]=0 .
$$

These follow from the GIG Lévy measure in (10) and $\beta=1 / 2$, which imply that $\int_{0}^{\infty} \bar{Q}(t) d t=\int_{0}^{\infty} t q(d t)=\frac{\kappa \gamma+1}{\gamma^{2}}$ and

$$
q(x)=\left[\frac{\kappa}{\pi \sqrt{2 x}} \Gamma(1 / 2)+\frac{1}{2}\right] \frac{1}{x} e^{-\gamma^{2} x / 2}, \quad x>0 .
$$

See Morales (2004) for details.

To obtain ruin probabilities, set $w(x, y)=1$ and $\delta=0$, which yields $\rho=0$ and the above $g$ and $h$ simplify to:

$$
g(x)=\frac{1}{1+\theta} \frac{\gamma^{2}}{(\kappa \gamma+1)}\left[\frac{\kappa \Gamma\left(\frac{1}{2}\right)}{\pi \sqrt{2}} \int_{x}^{\infty} t^{-\frac{3}{2}} e^{-\frac{\gamma^{2}}{2} t} d t+\frac{1}{2} \int_{x}^{\infty} t^{-1} e^{-\frac{\gamma^{2}}{2} t} d t\right]
$$

and

$$
\begin{aligned}
& h(x)=\frac{1-M(x)}{1+\theta} \\
& =\frac{1}{1+\theta} \frac{\gamma^{2}}{(\kappa \gamma+1)}\left\{\frac{2 \kappa \Gamma\left(\frac{1}{2}\right)}{\pi \gamma} \bar{\Gamma}\left(\frac{\gamma^{2}}{2} x ; \frac{1}{2}\right)-\frac{\kappa \Gamma\left(\frac{1}{2}\right)}{\pi}\left[\frac{2}{\gamma} \bar{\Gamma}\left(\frac{\gamma^{2}}{2} x ; \frac{3}{2}\right)\right.\right. \\
& \left.\left.-\gamma x \bar{\Gamma}\left(\frac{\gamma^{2}}{2} x ; \frac{1}{2}\right)\right]+\frac{1}{2}\left[\frac{2}{\gamma^{2}} e^{-\frac{\gamma^{2}}{2} x}-x \bar{\Gamma}\left(\frac{\gamma^{2}}{2} x ; 0\right)\right]\right\}, \quad x>0 \text {. }
\end{aligned}
$$


The G-S function then reduces to the ultimate ruin probability and the solution to (34) is consistent with the ruin function obtained in Section 3.2.5.

The discounted density $f_{\delta}(x, y \mid u)$ of a GIG with $\beta=1 / 2$ satisfies a renewal equation of the form in (37) with $g$ is given by (47) and

$$
\begin{aligned}
h(z)=\frac{1}{1+\theta} & e^{-\rho(x-z)} \frac{\gamma^{2}}{(\kappa \gamma+1)}\left[\frac{\kappa \Gamma(1 / 2)}{\pi \sqrt{2(y+x)}}+\frac{1}{2}\right] \\
& \times(x+y)^{-1} e^{-\gamma^{2}(x+y) / 2} \mathbb{I}_{\{x>z\}}(z), \quad z \geqslant 0 .
\end{aligned}
$$

By contrast, for parameter $\beta=-1 / 2$ (inverse Gaussian), these functions become

$$
g(z)=\frac{1}{1+\theta} \int_{z}^{\infty} e^{-\rho(y-z)} \frac{\gamma}{\kappa}\left[\frac{\kappa \Gamma(1 / 2)}{\pi \sqrt{2 y}}\right] y^{-1} e^{-\gamma^{2} y / 2} d y, \quad z \geq 0,
$$

and

$$
\begin{aligned}
h(z)=\frac{1}{1+\theta} & e^{-\rho(x-z)} \frac{\gamma}{\kappa}\left[\frac{\kappa \Gamma(1 / 2)}{\pi \sqrt{2(x+y)}}\right] \\
& \times(x+y)^{-1} e^{-\gamma^{2}(x+y) / 2} \mathbb{I}_{\{x>z\}}(z), \quad z \geqslant 0 .
\end{aligned}
$$

Finally, (38) and (39) imply that for the GIG process with $\beta=1 / 2$ :

$$
\begin{aligned}
f_{\delta, 2}(y \mid 0)=\frac{e^{\rho y}}{1+\theta} & \frac{\gamma^{2}}{(\kappa \gamma+1)}\left\{\frac { \sqrt { 2 } \kappa \Gamma ( 1 / 2 ) } { \pi } \left[y^{-1 / 2} e^{-\left(\gamma^{2} / 2+\rho\right) y}-\sqrt{\left(\gamma^{2} / 2+\rho\right)}\right.\right. \\
& \left.\left.\times \bar{\Gamma}\left(\left(\frac{\gamma^{2}}{2}+\rho\right) y ; \frac{1}{2}\right)\right]+\frac{1}{2} E_{1}\left(\left(\frac{\gamma^{2}}{2}+\rho\right) y\right)\right\}, \quad y>0
\end{aligned}
$$

while the corresponding discounted marginal of the surplus before ruin is given by

$$
\begin{aligned}
f_{1, \delta}(x \mid 0)= & \frac{1}{1+\theta} e^{-\rho x} m(x), \quad x>0, \\
= & \frac{e^{-\rho x}}{1+\theta} \frac{\gamma^{2}}{(\kappa \gamma+1)}\left\{\frac{2 \kappa \Gamma\left(\frac{1}{2}\right)}{\pi}\left[x^{-1 / 2} e^{-\gamma^{2} x / 2}-\frac{\gamma}{\sqrt{2}} \bar{\Gamma}\left(\frac{\gamma^{2}}{2} x ; \frac{1}{2}\right)\right]\right. \\
& \left.\quad+\frac{1}{2} E_{1}\left(\frac{\gamma^{2}}{2} y\right)\right\} .
\end{aligned}
$$


On the other hand, for the GIG with parameter $\beta=-1 / 2$ (inverse Gaussian) the last two expressions take the following slightly simpler forms:

$$
\begin{aligned}
f_{\delta, 2}(y \mid 0)= & \frac{e^{\rho y}}{1+\theta} \frac{\gamma}{\kappa}\left\{\frac { \sqrt { 2 } \kappa \Gamma ( 1 / 2 ) } { \pi } \left[y^{-1 / 2} e^{-\left(\gamma^{2} / 2+\rho\right) y}-\sqrt{\left(\gamma^{2} / 2+\rho\right)}\right.\right. \\
& \left.\left.\times \bar{\Gamma}\left(\left(\frac{\gamma^{2}}{2}+\rho\right) y ; \frac{1}{2}\right)\right]\right\}, \quad y>0,
\end{aligned}
$$

and

$$
\begin{aligned}
f_{1, \delta}(x \mid 0) & =\frac{1}{1+\theta} e^{-\rho x} m(x), \quad x>0 \\
& =\frac{e^{-\rho x}}{1+\theta} \frac{\gamma}{\kappa}\left\{\frac{2 \kappa \Gamma\left(\frac{1}{2}\right)}{\pi}\left[x^{-1 / 2} e^{-\gamma^{2} x / 2}-\frac{\gamma}{\sqrt{2}} \bar{\Gamma}\left(\frac{\gamma^{2}}{2} x ; \frac{1}{2}\right)\right]\right\} .
\end{aligned}
$$

Note that in these examples, when $\delta=0$ then $\rho=0$ also, and

$$
f_{1,0}(x \mid 0)=f_{0,2}(x \mid 0):=f(x \mid 0), \quad x>0,
$$

a consequence of the known duality property

$$
f(x, y \mid 0)=f(y, x \mid 0), \quad x, y>0 \text {. }
$$

It is clearly preserved here for risk processes driven by subordinators.

\section{$5 \quad$ Numerical examples}

This last section illustrates numerically some of the above results. In particular, expression (12) in Theorem 3.1 is used to compute ruin probabilities. It relates the Laplace transform of the ruin probability and the Laplace exponent of the aggregate claims process. Ruin probabilities are then obtained by numerical inversion of the Laplace transform for the model

$$
U(t)=u+c t-S(t), \quad t \geq 0
$$

when the aggregate claims process $S$ is gamma, inverse Gaussian and generalized inverse Gaussian process. 


\subsection{Gamma process}

Using (6) and (8) in (12), yields the Laplace transform of the ruin probability $\widehat{\psi}$ the gamma subordinator risk model:

$$
\widehat{\psi}(s)=\frac{1}{s}-\frac{c-a / b}{c s-a \ln (1+s / b)} .
$$

Recall that the process values $X(t)$ in Theorem 3.1 refer to $U(t)-u$ in risk model (1) and, therefore, $\mu_{X}=\mathbb{E}[X(1)]=\mathbb{E}[U(1)-u]=c-a / b$.

Using standard numerical inversion techniques on (49) produces the ruin probabilities illustrated in Table 1.

\subsection{Inverse Gaussian process}

The inverse Gaussian process is a particular case of the GIG when $\beta=-1 / 2$. Using (9) and (11), with this choice of $\beta$ in (12) yields the following Laplace transform of the ruin probability:

$$
\widehat{\psi}(s)=\frac{1}{s}-\frac{c-\kappa / \gamma}{c s+\kappa \gamma\left[1-\sqrt{1+2 s / \gamma^{2}}\right]} .
$$

Here $\mu_{X}=\mathbb{E}[X(1)]=\mathbb{E}[U(1)-u]=c-\kappa / \gamma$. We also use the fact that for $\beta= \pm 1 / 2$ the modified Bessel function $K_{\beta}$ simplifies to

$$
K_{-1 / 2}(x)=K_{1 / 2}(x)=\sqrt{\frac{\pi}{2}} x^{-1 / 2} e^{-x}
$$

Again, the values in Table 1 were obtained inverting (50) numerically.

\subsection{GIG process}

The generalized inverse Gaussian process also takes on a simpler form for $\beta=1 / 2$. Here $\mu_{X}=\mathbb{E}[X(1)]=\mathbb{E}[U(1)-u]=c-\frac{\kappa}{\gamma}\left[1+(\kappa \gamma)^{-1}\right]$. Using (51) and the fact that

$$
K_{1+1 / 2}(x)=\sqrt{\frac{\pi}{2}} x^{-1 / 2} e^{-x}\left[1+x^{-1}\right],
$$


the Laplace transform of the ruin probability $\psi$ becomes:

$$
\widehat{\psi}(s)=\frac{1}{s}-\frac{c-\frac{\kappa}{\gamma} \kappa / \gamma\left[1+(\kappa \gamma)^{-1}\right]}{c s+\kappa \gamma\left[1-\sqrt{1+2 s / \gamma^{2}}\right]-\ln \left[\sqrt{1+2 s / \gamma^{2}}\right]} .
$$

Table 1 also lists an illustrative example of the GIG process.

\subsection{Ruin probabilities}

Table 1 gives the ruin probability values in all three models above for different values of the loading $\theta$ and the initial surplus $u$. For comparison purposes all models illustrated have expected claims equal to 2 per time unit. The following parameters were used: Gamma process $[a=2.2, b=1.1]$, IG process $[\kappa=1.8, \gamma=0.9]$ and GIG process $[\beta=1 / 2, \kappa=1, \gamma=1]$.

\begin{tabular}{|c||c|c|c||c|c|c|}
\hline \multicolumn{1}{|c||}{} & \multicolumn{3}{c||}{$\theta=0.2$} & \multicolumn{3}{c|}{$\theta=0.5$} \\
\hline$u$ & Gamma & IG & GIG & Gamma & IG & GIG \\
\hline \hline 1 & 0.55525 & 0.57042 & 0.61963 & 0.30334 & 0.32773 & 0.37812 \\
\hline 2 & 0.39059 & 0.44236 & 0.49991 & 0.15644 & 0.20918 & 0.25466 \\
\hline 3 & 0.27592 & 0.34798 & 0.40685 & 0.08244 & 0.13846 & 0.17545 \\
\hline 4 & 0.19548 & 0.27537 & 0.33217 & 0.04405 & 0.09336 & 0.12224 \\
\hline 5 & 0.13894 & 0.21869 & 0.27167 & 0.02368 & 0.06373 & 0.08584 \\
\hline 6 & 0.09908 & 0.17413 & 0.22249 & 0.01266 & 0.04389 & 0.06063 \\
\hline 7 & 0.07086 & 0.13897 & 0.18245 & 0.00660 & 0.03041 & 0.04302 \\
\hline 8 & 0.05078 & 0.11114 & 0.14980 & 0.00325 & 0.02115 & 0.03061 \\
\hline 9 & 0.03641 & 0.08904 & 0.12315 & 0.00140 & 0.01473 & 0.02180 \\
\hline 10 & 0.02608 & 0.07145 & 0.10136 & 0.00039 & 0.01024 & 0.01552 \\
\hline
\end{tabular}

Table 1: Ruin probabilities for gamma $[a=2.2, b=1.1]$, inverse Gaussian $[\kappa=1.8, \gamma=0.9]$ and generalized inverse Gaussian $[\beta=1 / 2, \kappa=1, \gamma=1]$

For fixed expected aggregate claims, the gamma process is the least risky, while the GIG is the most risky, in terms of ultimate ruin probabilities.

This computational method for ruin probabilities can be used to obtain the figures in Dufresne et al. (1991), for the gamma process, and in Morales (2004) for the generalized inverse Gaussian process. Their parameters differ from those used here. Still our method and program reproduce their ruin probability values when the same parameters are used. 


\section{Conclusions}

We study the Gerber-Shiu function for a risk process driven by a subordinator, deriving tractable analytical expressions in some special cases, like the gamma, inverse Gaussian and generalized inverse Gaussian processes.

This modelling approach has the advantage of yielding a known distribution for the aggregate claims. It contrasts with the classical approach to risk modelling that specifies the individual claim severity distribution, requiring convolution processes to derive the resulting aggregate claims distribution.

One disadvantage of subordinator risk models is their implied infinite number of small claims. Still, the model remains mathematically tractable and small jumps can be interpreted as an extra source of variation. This is akin to other models in the literature ([13]-[15], [21], [25], [27]-[28] or [31]).

A unifying approach to risk modelling through Lévy processes brings new insight on some well known risk models. It also enlarges the class of risk processes that allow the computation of several ruin related quantities.

The fluctuation theory for spectrally negative Lévy processes has evolved in parallel to the study of $\mathrm{G}-\mathrm{S}$ functions. Although they intersect, one theory is not a subset of the other. For instance, the $\mathrm{G}-\mathrm{S}$ function is now available for some Sparre Andersen risk models that are not Lévy processes. Similarly, the theory of exit times for Lévy processes considers more general hitting times than the crossing of ruin or dividend barriers.

We provide here a review of classical ruin problems, as seen from a Lévy processes perspective. It gives two unifying computational methods for the ruin probability of any subordinator risk process and reproduces the known ruin probabilities in the gamma and GIG cases. One method is based on the solution of a renewal equation and requires evaluating series of convolutions. The other relies on the numerical inversion of Laplace transforms. Clearly, G$\mathrm{S}$ functions can be further explored with these examples to obtain moments for the time of ruin or asymptotic expressions for the probability of ruin or of overshoot.

The $\mathrm{G}-\mathrm{S}$ function for the general model in (1) remains an objective for future research. It requires a slight redefinition of the $\mathrm{G}-\mathrm{S}$ function, as in the perturbed model identifying which jumps represent claims is no longer clear. Another goal for future research is to explore the implications on $\mathrm{G}-\mathrm{S}$ functions of the latest findings in quintuple laws for Lévy processes. 


\section{References}

[1] Abramowitz, M. and Stegun, I. (1970). Handbook of Mathematical Functions With Formulas, Graphs and Mathematical Tables.

[2] Asmussen, S. (2000). Ruin Probabilities. Advanced Series on Statistical Science and Applied Probability. World Scientific.

[3] Barndorff-Nielsen, O.E. and Halgreen, C. (1977). Infinite divisibility of the hyperbolic and generalized inverse Gaussian distributions. Zeitschrift für Wahrscheinlichkeitstheorie und verwandte Gebiete. 38. pp. 439-455.

[4] Barndorff-Nielsen, O.E., Mikosh, T. and Resnick, S., ed. (2001). Lévy Processes-Theory and Applications. Birkäuser.

[5] Bertoin, J. (1996). Lévy Processes. Cambridge Tracts in Mathematics. 121. Cambridge University Press.

[6] Bertoin, J. and Doney, R.A. (1994). Cramér's estimate for Lévy processes. Statistics and Probability Letters. (21). pp. 363-365.

[7] Bowers, M., Gerber, H., Hickman, J., Jones, D. and Nesbitt, C. (1997) Actuarial Mathematics, 2nd edition, Society of Actuaries, Schaumburg.

[8] Chaubey, Y., Garrido, J. and Trudeau, S. (1998). On the computation of aggregate claims distributions: some new approximations. Insurance: Mathematics and Economics. 23. pp. 215-230.

[9] Chiu, S.N. and Yin, C. (2005) "Passage times for a spectrally negative Lévy process with applications to risk theory". Bernoulli. 11(3). pp. 511522.

[10] Drekic, S.; Stafford, J. E. and Willmot, G. E. (2004). Symbolic Calculation of the Moments of the Time of Ruin. Insurance: Mathematics and Economics. (34). pp. 109-120.

[11] Doney, R. A. and Kyprianou, A. E. (2005). Overshoots and Undershoots of Lévy Processes. To appear in Annals of Applied Probability.

[12] Dufresne, F. and Gerber, H. (1991). Risk theory for the compound Poisson process that is perturbed by diffusion. Insurance:Mathematics and Economics. 10. pp. 51-59. 
[13] Dufresne, F., Gerber, H.U. and Shiu, E.S.W. (1991). Risk theory with the gamma process. ASTIN Bulletin. 21(2). pp. 177-192.

[14] Furrer, H.J. (1998). Risk processes perturbed by a $\alpha$-stable Lévy motion. Scandinavian Actuarial Journal. (1). pp. 59-74.

[15] Furrer, H.J., Michna, Z. and Weron, A. (1997). Stable Lévy motion approximation in collective risk theory. Insurance: Mathematics and Economics. 20. pp. 97-114.

[16] Gerber, H.U. and Landry, B. (1998). On a discounted penalty at ruin in a jump-diffusion and the perpetual put option. Insurance: Mathematics and Economics. (22). pp. 263-276.

[17] Gerber, H.U. and Shiu, E.S.W. (1997). The joint distribution of the time of ruin, the surplus immediately before ruin, and the deficit at ruin. Insurance: Mathematics and Economics. 21. pp. 129-137.

[18] Gerber, H.U. and Shiu, E.S.W. (1998a). On the time value of ruin. North American Actuarial Journal. 2(1). pp. 48-78.

[19] Gerber, H.U. and Shiu, E.S.W. (1998b). Pricing perpetual options for jump processes. North American Actuarial Journal. 2(3). pp. 101-112.

[20] Grandell, J. (1991). Aspects of Risk Theory. Springer Series in Statistics. Springer-Verlag.

[21] Huzak, M.; Perman, M.; Sikic, H. and Vondracek, Z. (2004). Ruin probabilities and decompositions for general perturbed risk processes. The Annals of Applied Probability. (14)3. pp. 1378-1397.

[22] Janicki, A. and Weron, A. (1994). Simulation and Chaotic Behavior of $\alpha$-stable Stochastic Processes. Monographs and Textbooks in Pure and Applied Mathematics. 178. Dekker.

[23] Jørgensen, B. (1982). Statistical Properties of the Generalized Inverse Gaussian Distribution. Lecture Notes in Statistics. 9. Springer-Verlag.

[24] Kaas, R., Goovaerts, M., Dhaene, J. and Denuit, M. (2001). Modern Actuarial Risk Theory. Kluwer Academic Publishers. 
[25] Klüppelberg, C.; Kyprianou, A.E. and Maller, R.A. (2004). Ruin probabilities and overshoots for general Lévy insurance risk processes. The Annals of Applied Probability. (14)4. pp. 1766-1801.

[26] Madan, D.B., Carr, P. and Chang, E.C. (1998). The variance gamma process and option pricing. European Finance Review. 2. pp. 79-105.

[27] Morales, M. (2004). Risk theory with the generalized inverse Gaussian Lévy process. ASTIN Bulletin. (2). pp. 361-377.

[28] Morales, M. and Schoutens, W. (2003). "A risk model driven by Lévy processes." Applied Stochastic Models in Business and Industry. 19. pp. $147-167$.

[29] Politis, K. and Pitts, S. (2005). Approximations for the Deficit at Ruin and the Mean Ruin Time in the Classical Poisson Model. Working Paper. University of Cambridge, England.

[30] Sato, K.I. (1999). Lévy Processes and Infinitely Divisible Distributions. Cambridge University Press.

[31] Yang, H. and Zhang, L. (2001). Spectrally negative Lévy processes with applications in risk theory. Advances in Applied Probability. 33(1). pp. 281291.

[32] Zoloratev, M.V. (1964). "The first passage time of a level and the behavior at infinity for a class of processes with independent increments. Probability Theory and its Applications. 9(4). pp. 653-662.

\section{A Proofs}

\section{A.1 Proof of (31) and (32)}

Let $\phi$ be the discounted penalty function for the risk process in (30). $S$ is a subordinator and it has an infinite number of small jumps. However, jumps larger than a certain threshold form a compound Poisson process. Therefore we can construct, for any $\epsilon>0$, a sequence of classical risk processes $U_{\epsilon}$ as follows:

$$
U_{\epsilon}(t)=u+c_{\epsilon} t-S_{\epsilon}(t), \quad t \geq 0
$$


where $S_{\epsilon}$ is a compound Poisson process with jump density

$$
f_{\epsilon}(x)=\frac{q(x)}{\int_{\epsilon}^{\infty} q(t) d t} \mathbb{I}_{(\epsilon, \infty)}(x)
$$

and arrival rate

$$
\lambda_{\epsilon}=\int_{\epsilon}^{\infty} q(t) d t
$$

The loaded premium is given by

$$
c_{\epsilon}=(1+\theta) \lambda_{\epsilon} \mu_{\epsilon}=(1+\theta) \int_{\epsilon}^{\infty} t q(t) d t
$$

where $\mu_{\epsilon}$ is the mean of $f_{\epsilon}$. As $\epsilon \rightarrow 0$, the sequence of processes in (53) converges weakly to the process in (30). This follows from the fact that the $U_{\epsilon}$ are Lévy processes and their sequence of triplets converges to the triplet of the process $U$ in (30).

On the other hand, since the process in (53) is a classical risk process for any $\epsilon>0$, then its $\mathrm{G}-\mathrm{S}$ function $\phi_{\epsilon}$ takes the following form:

$$
\phi_{\epsilon}(u)=h_{\epsilon} * \sum_{k=0}^{\infty} g_{\epsilon}^{* k}(u), \quad u \geq 0
$$

where, from (31)

$$
g_{\epsilon}(x)=\frac{\lambda_{\epsilon}}{c_{\epsilon}} \int_{x}^{\infty} e^{-\rho_{\epsilon}(y-x)} \frac{q(y)}{\int_{\epsilon}^{\infty} q(t) d t} d y, \quad x \geq 0,
$$

and from $(32)$

$$
h_{\epsilon}(x)=\frac{\lambda_{\epsilon}}{c_{\epsilon}} \int_{x}^{\infty} \int_{0}^{\infty} e^{-\rho_{\epsilon}(z-x)} w(z, y) \frac{q(z+y)}{\int_{\epsilon}^{\infty} q(t) d t} d y d z, \quad x \geq 0 .
$$

The coefficient $\rho_{\epsilon}$ is the nonnegative solution of (33), which here is:

$$
-\delta+c r-\Psi_{\epsilon}(r)=0
$$

where $\Psi_{\epsilon}$ is the Laplace exponent of the compound Poisson process $S_{\epsilon}$ in (53).

As $\epsilon \rightarrow 0$, the $\mathrm{G}-\mathrm{S}$ function $\phi_{\epsilon} \rightarrow \phi$ and equations (54), (55), (56) and (57) converge to (29), (31), (32) and (33) respectively. 


\section{A.2 Proof of Proposition 4.1}

To verify (35) we first need the following result.

Theorem A.1 Let $X_{t}$ be a subordinator with Lévy triplet $(a, 0, q)$. Define the $\varrho$-Esscher transform of $X$ as the one induced by the density process $\frac{\exp \left(\varrho X_{t}\right)}{\chi_{t}(\varrho)}$ where $\chi_{t}$ is the characteristic function of the process. Then, the characteristic function $\chi_{t}^{\varrho}$ of the transformed process $X_{t}^{\varrho}$ can be written in terms of the original characteristic function $\chi_{t}$ as follows:

$$
\chi_{t}^{\varrho}(z)=\frac{\chi(z-i \varrho)}{\chi_{t}(-i \varrho)} .
$$

Moreover, the process $X_{t}^{\varrho}$ is a Lévy process with triplet $\left(a_{\varrho}=a, 0, q_{\varrho}(d x)=\right.$ $\left.e^{\varrho x} q(d x)\right)$.

This result can be found in Morales and Schoutens (2003), or in standard references on Lévy processes such as Sato (1999) or Bertoin (1996).

Now, from (29) we have that the Laplace transform of $\phi$ can be written as

$$
\widehat{\phi}(s)=\widehat{h}(s) \sum_{k=0}^{\infty} \widehat{g}^{k}(s)=\frac{\widehat{h}(s)}{1-\widehat{g}(s)} .
$$

On the other hand, from (31) we have the Laplace transform of $g$ :

$$
\widehat{g}(s)=\frac{1}{1+\theta} \int_{0}^{\infty} e^{-(s-\rho) x} \int_{x}^{\infty} e^{-\rho y} \frac{q(y)}{\int_{0}^{\infty} \bar{Q}(t) d t} d y d x .
$$

From Theorem A.1, we see that $e^{-\rho y} q(d y)$ is the Lévy measure $q_{\rho}$ of a $-\rho$ Esscher transformed process. Hence $\widehat{g}(s)$ can be rewritten as

$$
\widehat{g}(s)=\frac{1}{1+\theta} \int_{0}^{\infty} e^{-(s-\rho) x} \frac{\mu_{\rho}}{\mu_{S}} \int_{x}^{\infty} \frac{q_{\rho}(y)}{\int_{0}^{\infty} \bar{Q}_{\rho}(t) d t} d y d x,
$$

where $\mu_{\rho}=\mathbb{E}\left[S_{\rho}(1)\right]=\int_{0}^{\infty} \bar{Q}_{\rho}(t) d t=\int_{0}^{\infty} t q_{\rho}(t) d t$. The latter can be rewritten as

$$
\mu_{\rho}=\mu_{S}+\int_{0}^{\infty} x\left(e^{-\rho x}-1\right) q(d x) .
$$

From (59) we can identify $\frac{q_{\rho}(y)}{\int_{0}^{\infty} Q_{\rho}(t) d t}$ as the ladder-height density $m_{\rho}$ of a risk process driven by $S_{\rho}$. Therefore, we can write (59) as

$$
\widehat{g}(s)=\frac{1}{1+\theta} \int_{0}^{\infty} e^{-(s-\rho) x} \frac{\mu_{\rho}}{\mu_{S}} \int_{x}^{\infty} m_{\rho}(y) d y d x=\frac{1}{1+\theta} \frac{\mu_{\rho}}{\mu_{S}} \xi_{M_{\rho}}(s-\rho),
$$


where $\xi_{M_{\rho}}$ is the Laplace transform of the density $m_{\rho}$. Substituting this last expression into (58) gives

$$
\widehat{\phi}(s)=\frac{\widehat{h}(u)}{1-\frac{1}{1+\theta} \frac{\mu_{\rho}}{\mu_{S}} \xi_{M_{\rho}}(s-\rho)} .
$$

Letting $\theta_{\rho}=\frac{(1+\theta) \mu_{S}}{\mu_{\rho}}-1$, then multiplying and dividing by $\frac{\theta_{\rho}}{1+\theta_{\rho}}$ gives (35). 


\section{List of Recent Technical Reports}

46. Shuanming Li and José Garrido, On the Time Value of Ruin for a Sparre Anderson Risk Process Perturbed by Diffusion, November 2003

47. Yogendra P. Chaubey, Cynthia M. DeSouza and Fassil Nebebe, Bayesian Inference for Small Area Estimation under the Inverse Gaussian Model via Cibbs Sampling, December 2003

48. Alexander Melnikov and Victoria Skornyakova, Pricing of EquityLinked Life Insurance Contracts with Flexible Guarantees, May 2004

49. Yi Lu and José Garrido, Regime-Switching Periodic Models for Claim Counts, June 2004.

50. I. Urrutia-Romaní, R. Rodríguez-Ramos, J. Bravo-Castillero and R. Guinovart-Díaz, Asymptotic Homogenization Method Applied to Linear Viscoelastic Composites. Examples, August 2004.

51. Yi Lu and José Garrido, Double Periodic Non-Homogeneous Poisson Models for Hurricanes Data, September 2004.

52. M.I. Beg and M. Ahsanullah, On Characterizing Distributions by Conditional Expectations of Functions of Generalized Order Statistics, September, 2004.

53. M.I. Beg and M. Ahsanullah, Concomitants of Generalized Order Statistics from Farlie-Gumbel-Morgenstern Distributions, September, 2004.

54. Yogendra P. Chaubey and Debaraj Sen, An investigation into properties of an estimator of mean of an inverse Gaussian population, September, 2004.

55. Steven N. Evans and Xiaowen Zhou, Balls-in-boxes duality for coalescing random walks and coalescing Brownian motions, September, 2004.

56. Qihe Tang, Asymptotic ruin probabilities of the renewal model with constant interest force and regular variation, November, 2004.

57. Xiaowen Zhou, On a classical risk model with a constant dividend barrier, November, 2004. 
58. K. Balasubramanian and M.I. Beg, Three isomorphic vector spaces-II, December, 2004.

59. Michael A. Kouritzin and Wei Sun, Rates for branching particle approximations of continuous-discrete filters, December, 2004.

60. Rob Kaas and Qihe Tang, Introducing a dependence structure to the occurrences in studying precise large deviations for the total claim amount , December, 2004.

61. Qihe Tang and Gurami Tsitsiashvili, Finite and infinite time ruin probabilities in the presence of stochastic returns on investments, December, 2004.

62. Alexander Melnikov and Victoria Skornyakova, Efficient hedging methodology applied to equity-linked life insurance, February, 2005.

63. Qihe Tang, The finite time ruin probability of the compound Poisson model with constant interest force, June, 2005.

64. Marc J. Goovaerts, Rob Kaas, Roger J.A. Laeven, Qihe Tang and Raluca Vernic, The tail probability of discounted sums of Pareto-like losses in insurance, August, 2005.

65. Yogendra P. Chaubey and Haipeng Xu, Smooth estimation of survival functions under mean residual life ordering, August, 2005.

66. Xiaowen Zhou, Stepping-stone model with circular Brownian migration, August, 2005.

67. José Garrido and Manuel Morales, On the expected discounted penalty function for Lévy risk processes, November, 2005.

Copies of technical reports can be requested from:

Prof. Xiaowen Zhou

Department of Mathematics and Statistics

Concordia University

7141, Sherbrooke Street West

Montréal (QC) H4B 1R6 CANADA 